Editorial

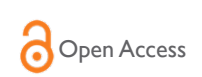

CrossMark

\title{
Personalized gene therapy proposal
}

\section{Editorial}

Gene therapy is a kind of treatment which involves delivering genetic material into patients' cells to fight or prevent disease. Several gene therapy objectives are being investigated, including:
i. Replacing an abnormal gene with a normal gene;
ii. Inactivating or knocking out a mutated gene;
iii. Introducing a new gene for fighting a disease.

Somatic gene therapy, which is viewed as a more conservative, safer approach, can be split into two categories: Ex vivo, in which the patients' cells are modified outside the body and then transplanted back into patients' bodies again. In vivo, in which the therapeutic genes are transferred to cells inside the patients' bodies. Gene therapy can be used for three types of diseases:

i. Monogenic disorders, for example: Sickle Cell Anemia, Hemophilia, Duchenne Muscular Dystrophy, Huntington's Disease, Familial Parkinson's, Hypercholesterolemia, and so on;

ii. Polygenic disorders, such as Heart Disease, Cancer, Diabetes, Schizophrenia and Alzheimer's disease;

iii. Infectious diseases, such as HIV.

The technology is still in its infancy, but it has been used with some success. 2,210 clinical trials have been approved in worldwide from 1989 to 2015. On September 14, 1990, the first approved gene therapy procedure was carried out on a 4year old girl, Ashanti DeSilva, with Adenosine Deaminase Deficiency (ADA). Although this procedure was not a cure, the therapy needs to be repeated every two years. She attended college and now she is still in good health with a normal life. In 2003, the first commercial gene therapy medicine (Gendicine) carrying the p53 tumor-suppressor gene was available on the market in China. In November 2012, the European Commission approved the gene therapy Glybera, which is used to treat Familial Hyperchylomicronemia. This is the first gene therapy
Volume 2 Issue I - 2016

\section{Bin Li}

Department of Biochemistry and Molecular \& Cellular Biology, Georgetown University Medical Center, USA

Correspondence: Bin Li, Department of Biochemistry and Molecular \& Cellular Biology, Georgetown University Medical Center, USA, Tel 2026870273, Email bin.li@georgetown.edu

Received: January 06, 2016 | Published: January 07, 2016

drug approved by regulatory authorities in the Western world. In 2015, a baby girl with acute Lymphoblastic Leukemia was cured by gene-edited T-cells in the United Kingdom. Personalized medicine (PM) is a medical model in which the genetic information plays a major role for the customization of healthcare. Personalized Gene Therapy is the most cutting-edge treatment method, especially for monogenic disorders and cancer. Most monogenic disorders only have very rare cases; however they have variable mutations for single genes. Additionally, different cancer cells also involve a large amount of genetic variations. On the other side, since the gene-edited cells are the patients' own cells, they don't have to be additionally modified to prevent graft-versus-host disease. Therefore, the personalized gene therapy will be a new way to cure many currently incurable diseases, such as monogenic disorders and cancer.

\section{Acknowledgements}

None.

\section{Conflict of interest}

Author declares that there is no conflict of interest. 